EMITTER International Journal of Engineering Technology ISSN: 2443-1168, Vol. 8, No. 2, December 2020, pp. 459 476

DOI : 10.24003 /emitter.v8i2.566

\title{
Perfomance Comparison of Genetic and Greedy Algorithms in Underlay Device-to-Device Communication
}

\author{
Salma Pratiwi ${ }^{1}$, Arfianto Fahmi ${ }^{2}$, Vinsensius Sigit Widhi Prabowo ${ }^{3}$ \\ School of Electrical Engineering \\ Telkom University, Bandung \\ E-mail: salmaprtw@student.telkomuniversity.ac.id ${ }^{1}$, \\ arfiantof@telkomuniversity.ac.id ${ }^{2}$, vinsensiusvsw@telkomuniversity.ac.id ${ }^{3}$ \\ Received September 14, 2020; Revised October 16, 2020; Accepted December 9, 2020
}

\begin{abstract}
The number of mobile devices continues to grow across the world and impacts the overall network load. An increasing number of devices that connected to the main network will harm the system's quality of services. To solve this problem, LTE-A technology introduce the Device-to-Device (D2D) Communication. D2D enables direct communications between users without routing via eNB, so the network load could be maintained. D2D scheme also can be underlayed on an existing cell using same resource with the conventional cellular users (CU). A good resource allocation algorithm is needed to achieve a proper quality of services and to maintain the interference level between D2D users and CUs. This works tries to analyze the performances of Genetics based resource allocation algorithm and then compared it with the conventional greedy algorithm. In general, Genetic algorithm has three steps to allocates resource block (RB) to each user, proportional selection, crossover, and mutation, and these three steps executed repeatedly to achieve the best performances. From the simulation result, Genetic algorithm can achieve better energy efficiency, lower usage power, and lower interference level. Altough genetic algorithm caused lower system's sumrate and spectral efficiency.
\end{abstract}

Keywords: Device-to-Device (D2D), Genetic Algorithm, Spectral Efficiency, Energy Efficiency, Interference Mitigation.

\section{INTRODUCTION}

The number of mobile communication users continues to increase all over the world. This raises the problem of increasingly crowded communication traffic in cellular networks. One solution to this problem is the Device-to-Device (D2D) communication technology. LTE enables the usage of heterogeneous network in the system such as Femtocells and D2D communications [1]. D2D communication as a technology component for LTEA enables a direct connection between devices, without rerouting the traffic through any network infrastructure. Under normal conditions, the network 
controls the usage of direct links radio resource minimize the level of interference. This technology promises to increase coverage, offload backhaul, provide fallback connectivity, and increase spectrum utilization and capacity per area [2]. D2D communication has two types based on the used spectrum by D2D users and their impact on cellular users, which are inband and outband D2D communication [3].

Outband D2D communications exploit the unlicensed spectrum. The advantages of this scheme are the interference between D2D links and cellular links is minimum, since D2D communications use license-exempt bands, similar to Wi-Fi Direct, Wi-Fi, Bluetooth, and Ultra-Wideband technologies.

In the inband scheme, D2D communications communicate through a licensed spectrum, which is also being used by conventional cellular users. There are two types of inband scheme, underlay D2D communications and overlay D2D communications. Overlay scheme allocates dedicated links for D2D users, meanwhile in underlay scheme, D2D user utilize the same spectrum with some cellular users.

Overlay scheme offers a good system performance, since D2D users can communicate without co-channel interference on dedicated resources, but will have a low spectral efficiency. On the other hand, underlay scheme share the same spectrum resources with some other cellular user equipments (CUEs). With spectrum sharing, D2D communications can improve spectrum efficiency and network throughput which are two important performance indices. Nevertheless, mutual interference between D2D user equipments (DUEs) and CUEs could critically affect the system performance [3]. Thus, a good resource allocation algorithm to allocate radio resources properly is needed to maintain the system performances.

\section{RELATED WORKS}

There are several research related to resource allocation in underlay D2D communication [4-7]. In [4], the research analyzes system performance based on heuristic algorithm, then compares it with minimum interference algorithm and random allocation algorithm. The study presented a simulation result of a heuristic algorithm working optimally when the system has a dense number of D2D pairs.

Work [5] proposed a joint mode selection allocation algorithm. The research developed a complex branch-and-bound method to have low complexity for D2D mode selection and channel allocations problems. This works proposed two steps allocation process, which are power control and channel determination that executed independently.

Work [6] proposed resources allocation based on Genetic algorithms to minimize the interference level among users, maximize the spectral efficiency, and maintain the value of Signal to Interference plus Noise Ratio (SINR) of cellular users due to D2D communication. That study used the channel prediction method and evolves toward global maximum by searching different parts of search space simultaneously. 
In [7], process allocation of resource in uplink way with D2D pair used Join Greedy algorithm, then compares it with the conventional greedy algorithm. Based on the simulation results, the joint greedy allocation scheme achieve better efficiency and fairness compared with greedy allocation algorithm.

This works analyze and compare the performance parameters between Genetic and greedy algorithm on D2D communication systems, including system interference, system sum-rate, system spectral efficiency, total energy system, and system energy efficiency. The rest of paper is composed as follows. In Section 3, the concepts those can solve the problem stated in the Introduction is explained. The system model is described in Section 4. Numerical simulation results are presented in Section 5. Finally, the conclusions of the paper are given in Section 6.

\section{ORIGINALITY}

This works tries to modify the genetic based algorithm in [6] and compared with the greedy algorithm. The modification lies in the structure of the two main process of algorithm, which are the mutation and the crossover. The genetic based algorithm has no restriction on a number of D2D pairs that can use same RB with cellular user in an instance, but a minimum value of SINR is required. Genetic based algorithm is being used as a comparison. This works tries to evaluate the genetics algorithm performances in several parameters, such as spectral efficiency, energy efficiency, total power usage, interference level, and the system's sumrate. This works done to give a better explanation about the performances of genetics allocation algorithm.

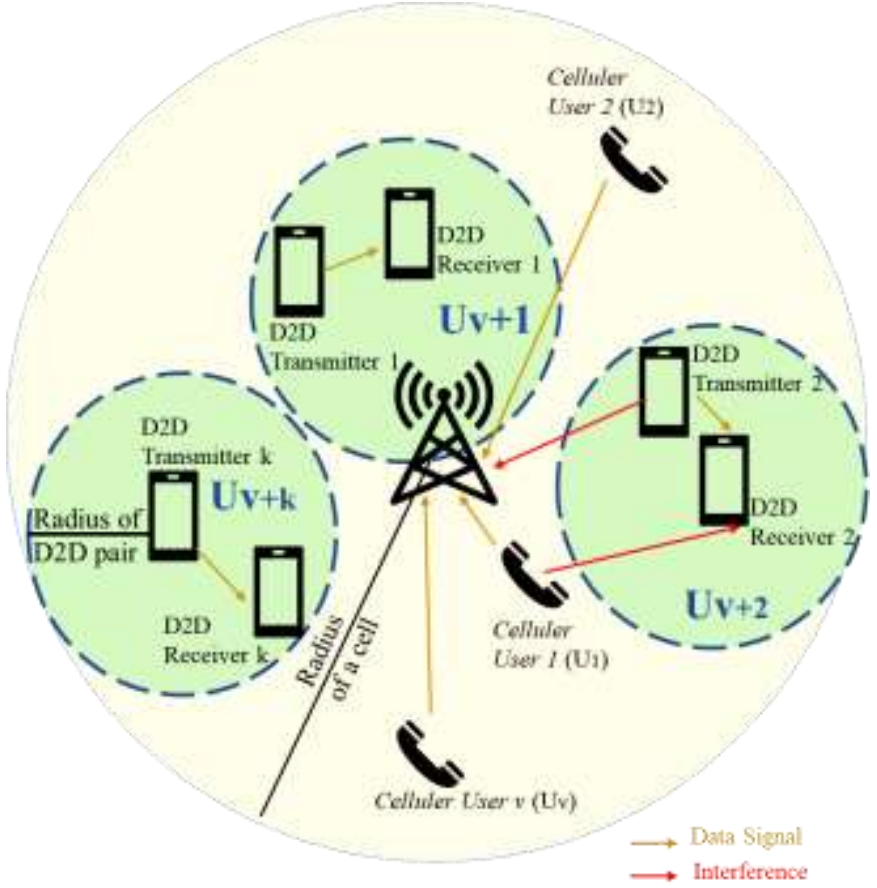

Figure 1. The model systemAn example of an interference graph in D2D communication 


\section{SYSTEM DESIGN}

System model that being used in this research is a single cell with one eNB in the center. There are two type of user, which are cellular users (CUEs) that communicates through eNB, and D2D users (DUEs) which can communicates directly between device and form a DUE pairs. These users are randomly distributed on cell area. The cell model can be seen on Figure 1

The pair of communications were defined as $\mathrm{N}=\left\{U_{1}, U_{2}, \ldots, U_{V}, U_{V+1}, \ldots\right.$ $\left.U_{V+K}\right\}$, where $U_{\mathrm{i}}$ showed the $i^{\text {th }}$ communication, then $V$ and $K$ is the total number of CUEs and DUE pairs respectively. The set of CUEs is noted by $M=\left\{U_{1}, U_{2}, \ldots\right.$, $\left.U_{V}\right\}$, and the set of D2D pairs was defined by $\mathrm{D}=\left\{U_{V+1}, U_{V+2}, \ldots U_{V+\mathrm{K}}\right\}$. The set of resources block (RB) denoted by $\mathrm{R}=\left\{R B_{1}, R B_{2}, \ldots, R B_{Q}\right\}$. Where $Q$ denotes the total number of RBs. This model system used a matrix with size $Q x(V+K)$ with an element of $a_{\mathrm{r}, \mathrm{i}}$ an element of $a_{\mathrm{r}, \mathrm{I}}=1$ if $i^{\text {th }}$ user utilizes the $r^{\text {th }}$ of RB, and $a_{\mathrm{r}, \mathrm{I}}=$ 0 if $i^{\text {th }}$ user did not utilize the $r^{\text {th }}$ of RB [6].

Figure 2 shows an example of an interference graph in D2D communication. Nodes represent communication, either cellular or D2D communication. The edges were interference between two connected nodes. Let $U_{1}, U_{3}$, and $U_{4}$ were in the same $R B$, except $U_{2}$. First cellular user $\left(U_{1}\right)$ will be affected by interference from the D2D users ( $U_{3}$ and $\left.U_{4}\right)$, as well as $U_{3}$ will be affected by interference from $U_{1}$. $\mathrm{I}_{\mathrm{i}, \mathrm{j}, \mathrm{r}}$ was the edge weight which indicated the set of interference value from $\mathrm{i}^{\text {th }}$ node to $\mathrm{j}^{\text {th }}$ node at $\mathrm{r}^{\text {th }}$ resource block. Since $\mathrm{U}_{1}$ 's and $\mathrm{U}_{2}$ 's receiver is the eNB, if they shared a RB the receiver could not differentiate the signals. Thus, there was no interference between cellular users.

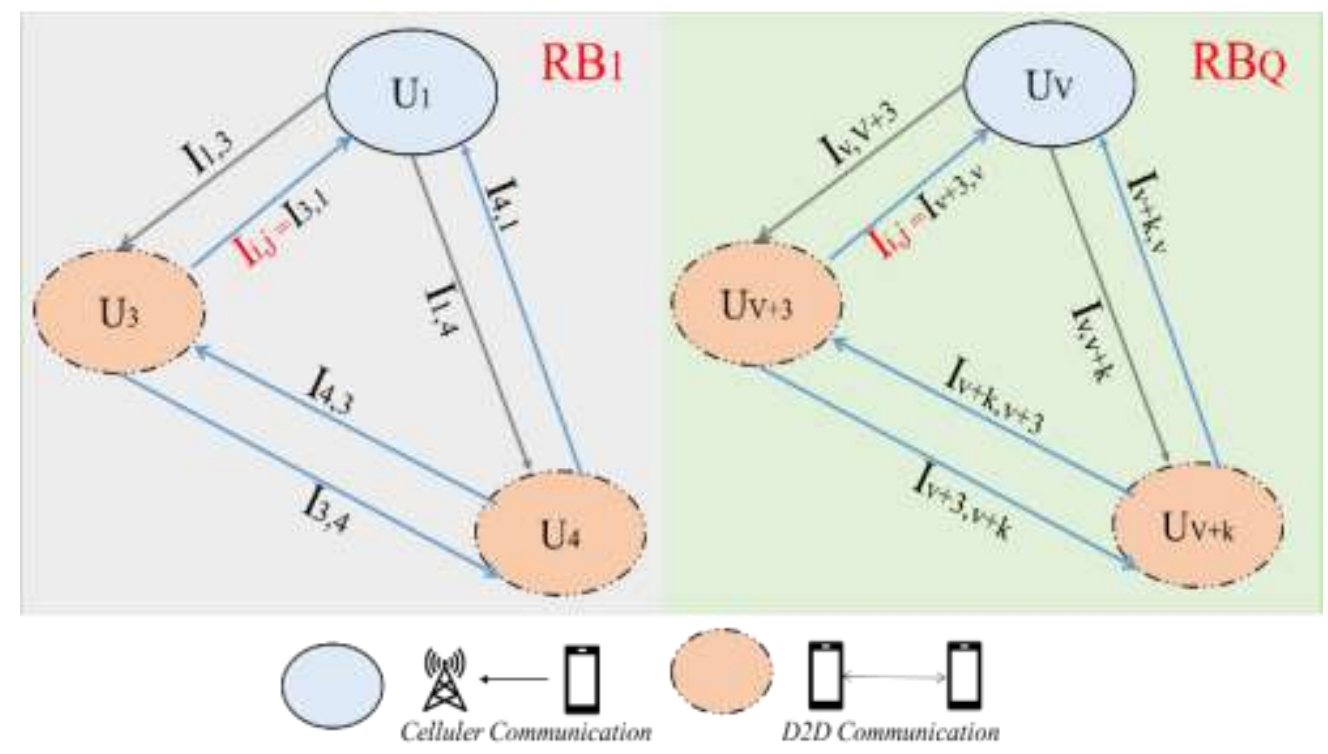

(b)

Figure 2. An example of an interference graph in D2D communication. 
Maximum spectral efficiency of $i^{\text {th }}$ communication at $r^{\text {th }} \mathrm{RB}$, either a CUE or a DUE pair, can be calculated by:

$$
S e_{i, r}=\frac{B \times \log _{2}\left(1+\operatorname{SIN} R_{i, r}\right)}{B S}
$$

where B is the bandwidth of the RB and Bs is the system bandwidth in Hertz, SINR is the signal to interference plus noise ratio. The $\operatorname{SINR}_{\mathrm{i}, \mathrm{r}}$ can be calculated by [6] :

$$
\begin{aligned}
& \operatorname{SINR}_{i, r}=\frac{a_{i, r} \cdot p_{i, r} \cdot g_{i, i, r}}{\sigma^{2}+I_{i}^{r}} \\
& I_{i}^{r}=\sum_{j=1, j \neq i}^{V+K} a_{j, r} \cdot I_{i}^{r}=\sum_{j=1, j \neq i}^{V+K} a_{j, r} \cdot p_{j, r} \cdot g_{j, i, r} \\
& \text { Se }_{\text {network }}=\sum_{i=1}^{V+K} \sum_{r=1}^{Q} \log _{2}\left(1+\operatorname{SINR}_{i, r}\right) . \\
& \text { subject to }\left\{\begin{array}{cc}
C_{1}: \sum_{i=1}^{V} a_{i, r} \leq 1, & i \in M, \forall_{r} \in R \\
C_{2}: p_{i}<p_{d m a x}, & i \in D \\
C_{3}: p_{i}<p_{\text {cmax }}, & i \in M \\
C_{4}: \operatorname{SINR}_{i, r} \geq \operatorname{SINR}_{\min }, & i \in M, \forall_{r} \in R
\end{array}\right.
\end{aligned}
$$

In equation $2, \mathrm{p}_{\mathrm{i}, \mathrm{r}}$ is the transmission power of the $\mathrm{i}^{\text {th }}$ node at $^{\mathrm{th}} \mathrm{RB}, \mathrm{g}_{\mathrm{i}, \mathrm{j}, \mathrm{r}}$, is the channel gain between the transmitter of the $i^{\text {th }}$ node and receiver of the $j^{\text {th }}$ node at $\mathrm{r}^{\text {th }} \mathrm{RB}$ and $\sigma^{2}$ is the noise power. $I_{i}^{r}$ is the total interference from other nodes to $i^{\text {th }}$ node which can be written on equation 3. Equation 4 defines the network's spectral efficiency. Thus, the maximization problem defines in equation 5 [6]. $C_{1}$ make sure there will be at least 1 user on each RB. $C_{2}$ and $C_{3}$ limit the transmission power of each user. $p_{d m a x}$ and $p_{c m a x}$ are maximum transmission power of D2D users and cellular users, respectively. $\mathrm{C}_{4}$ shows that SINRmin is a minimum SINR for cellular users. The other simulation parameters can be seen on Table 1 .

Table 1. Channel classification in mobile communication [6-8]

\begin{tabular}{|c|c|}
\hline System Characteristics & Channel type \\
\hline Cell radius & $500 \mathrm{~m}$ \\
\hline Channel model & COST-231 Hata model \\
\hline Fading & Rayleigh \\
\hline Shadowing & $1.8 \mathrm{GHz}$ \\
\hline Carrier frequency & $180 \mathrm{KHz}$ \\
\hline Bandwidth of RBs & $10 \mathrm{~m}$ \\
\hline Maximum D2D pairs distance & $23 \mathrm{dBm}$ \\
\hline Maximum power of DUE & $23 \mathrm{dBm}$ \\
\hline Maximum power of CUE & $-40 \mathrm{dBm}$ \\
\hline Minimum power of DUE & $-174 \mathrm{dBm} / \mathrm{Hz}$ \\
\hline Noise power density & \\
\hline
\end{tabular}




\subsection{Genetic Algorithm}

The genetic algorithm consists of 3 main phases to form a new generation based on the current generation: proportional selection, crossover, and mutation. The algorithm started with a set of candidate solutions (chromosomes), which use dedicated power and RBs to communicate. Chromosomes should satisfy the constraint of the optimization problem in equation (5). The set of chromosomes was called a generation, that could be changed iteratively. Parents would be selected among the current generation to produce children (offspring) to form next generation. Parents will be selected based on the fitness of each chromosome, in terms of the spectral efficiency. After several iterations, the generation will acheve optimum value [6].

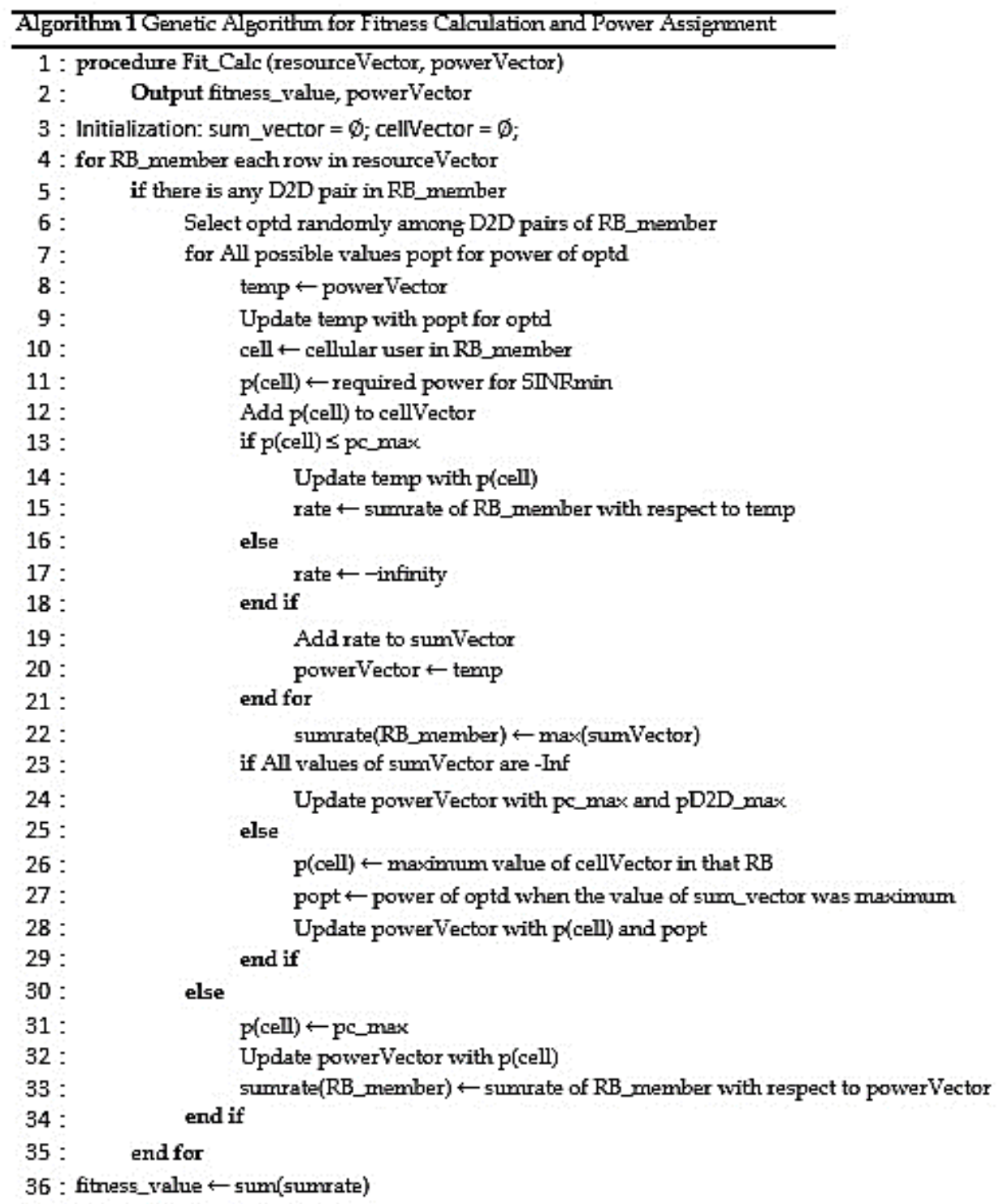

Figure 2. Genetic algorithm for fitness and power assignment 


\subsubsection{Fitness Function and Power Assignment Calculation}

The fitness function calculates the value of a chromosome. Since the purposes of the algorithm was to maximize the spectral efficiency of the network, so the fitness value of a chromosome equals to spectral efficiency of network according to equation (4). On each iteration, power of the users will be optimized. On each iteration, a random D2D communication (if any unnalocated DUE) will be selected by each RB. The SINR of CUE on that corresponding RB will be set to SINRmin. Then, the power of $i^{\text {th }}$ cellular user at $\mathrm{r}^{\text {th }} \mathrm{RB}$ was calculated based on:

$$
p_{i, r}=S I N R_{\min } \times \frac{\sigma^{2}+\sum_{j=1, j \neq i}^{V+K} a_{j, r} \cdot p_{j, r} \cdot g_{j, i, r}}{a_{i, r} g_{i, i, r}}
$$

However, if an RB was used by only one CUE, then the transmission power of that CUE will be maximum. The pseudocode for fitness calculation and power assignment can be seen on Figure 2. Chromosome (in this case is resource allocation matrix and transmission power vector), is being used as the inputs of the algorithm.

\subsubsection{Proportional Selection}

The proportional selection process selects chromosomes to become parents for next generation, which have the best fitness value in terms of spectral efficiency. If fitness value of the chromosome $\mathrm{x}$ denoted by $\mathrm{f}_{\text {chromosome }}(\mathrm{x})$. The sets of fit $(\mathrm{t})$ which is the set of fitness values at $\mathrm{t}^{\text {th }}$ generation is denoted by [6]:

$$
f i t(t)=\left\{f_{\text {chrosome }}\left(x_{1}\right), f_{\text {chrosome }}\left(x_{2}\right), \ldots, f_{\text {chrosome }}\left(x_{N t}\right)\right\}
$$

where $\mathrm{x}_{\mathrm{i}}$ and $\mathrm{N}_{\mathrm{t}}$ are the $\mathrm{i}^{\text {th }}$ chromosome and the total number of chromosomes in $t^{\text {th }}$ generation, respectively.

\subsubsection{Crossover Process}

The crossover process will combine two "parents" and produces next generation's offspring. On the initial stages, a random length of offspring is given to a RB. The length is equal to the total possible communications that can be supported in that RB. Next step is selecting a random number in range $[1, \mathrm{~V}+\mathrm{K}]$ to be the crossover point (CV), which define the number of communication that have been taken from the parents. For example, $\mathrm{CP}=3$ mean there are 3 communications (including the power transmit on that communications) are taken from parent1, and the rest of the length will be taken from parent 2. All detailed algorithm can be seen on Figure 3, the illustration can be seen on Figure 4. This process will create a local optimum solution, which will be the reference for the mutation process. 

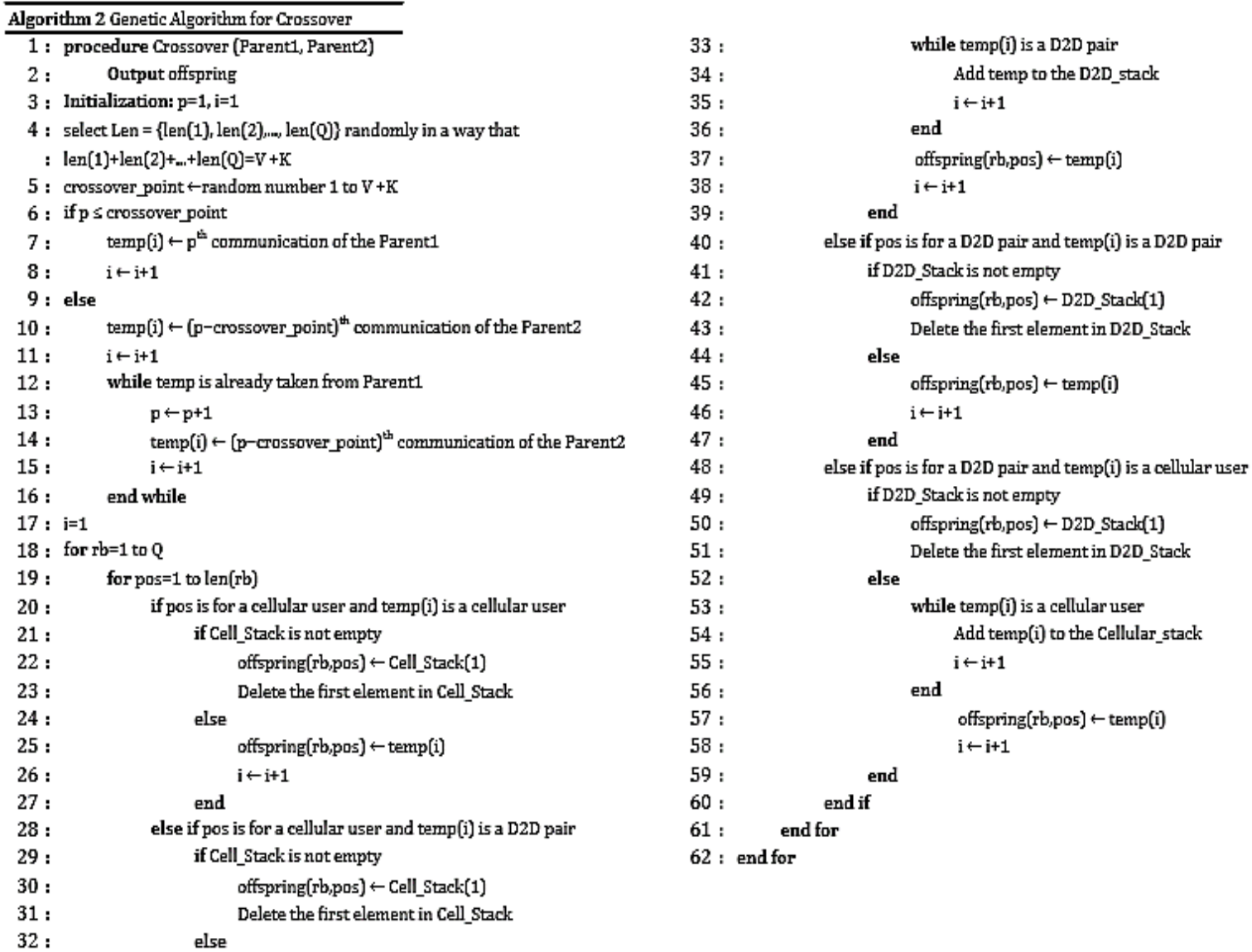

Figure 3. Algorithm 2 genetic algorithm for crossover

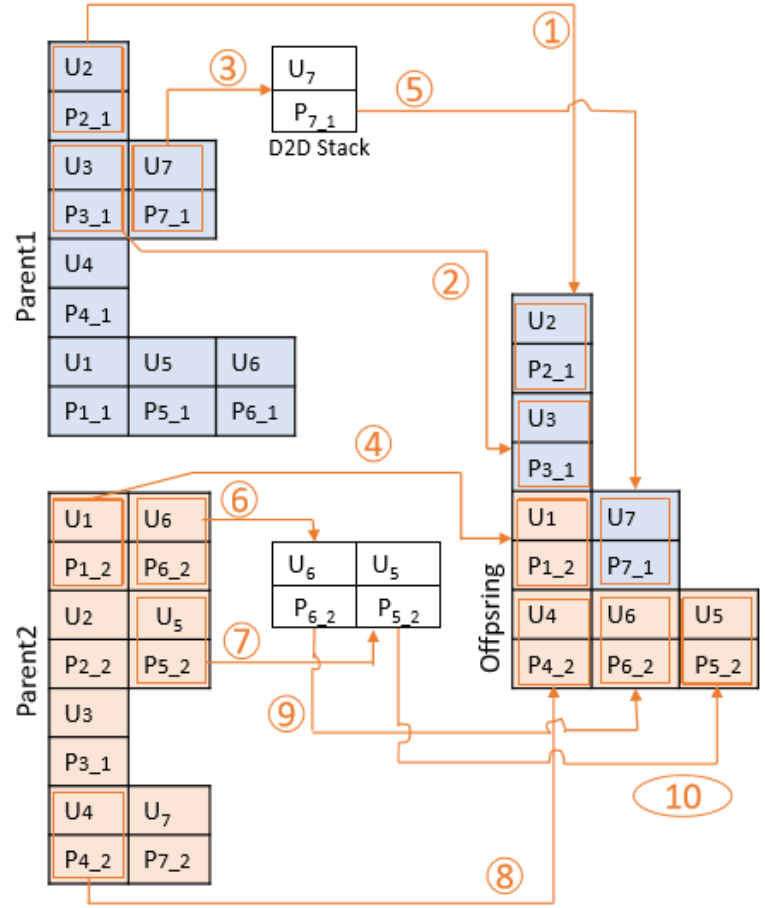

Figure 4. Crossover operation with $\mathrm{CP}$ is 3 


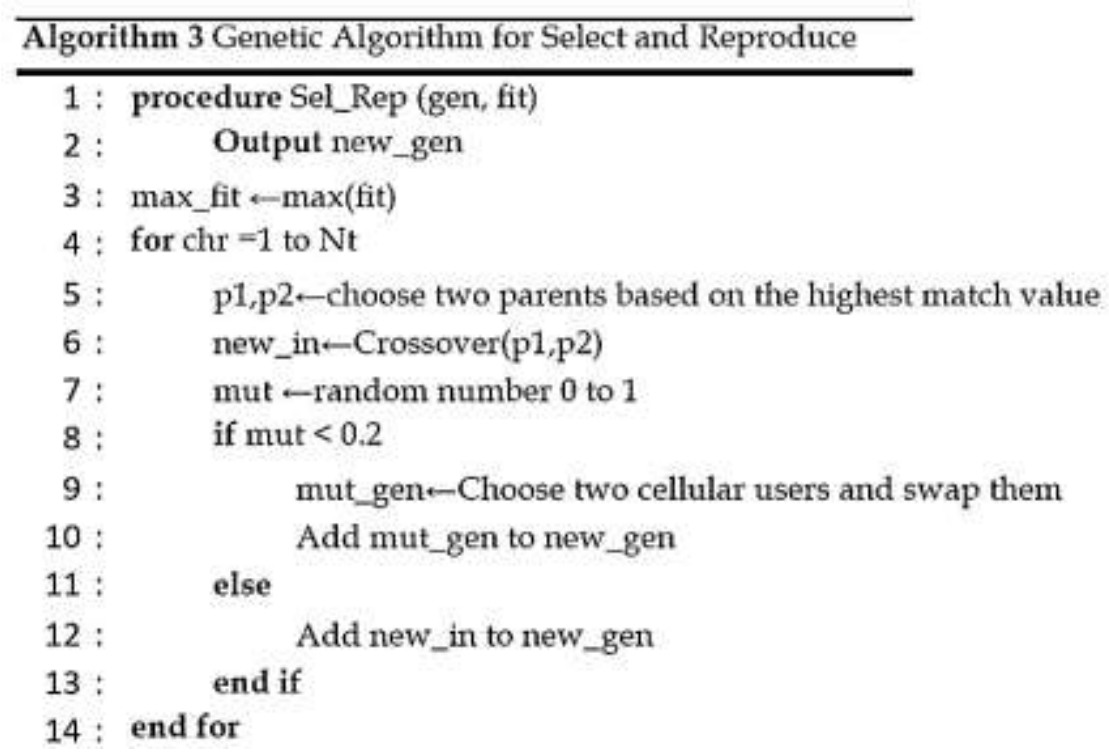

Figure 5. Genetic algorithm to select and reproduce

\subsubsection{Mutation}

The next process is mutation. Mutation is a random change on the chromosome with low probability to escape from local optimum solution [9]. This random change is applied by swapping two random DUEs on corresponding RB. To prevent random search, the probability of mutation must be low. Combination of three main process proportional selection, crossover operation and mutation were called select and reproduce, and can be seen on Figure 5.

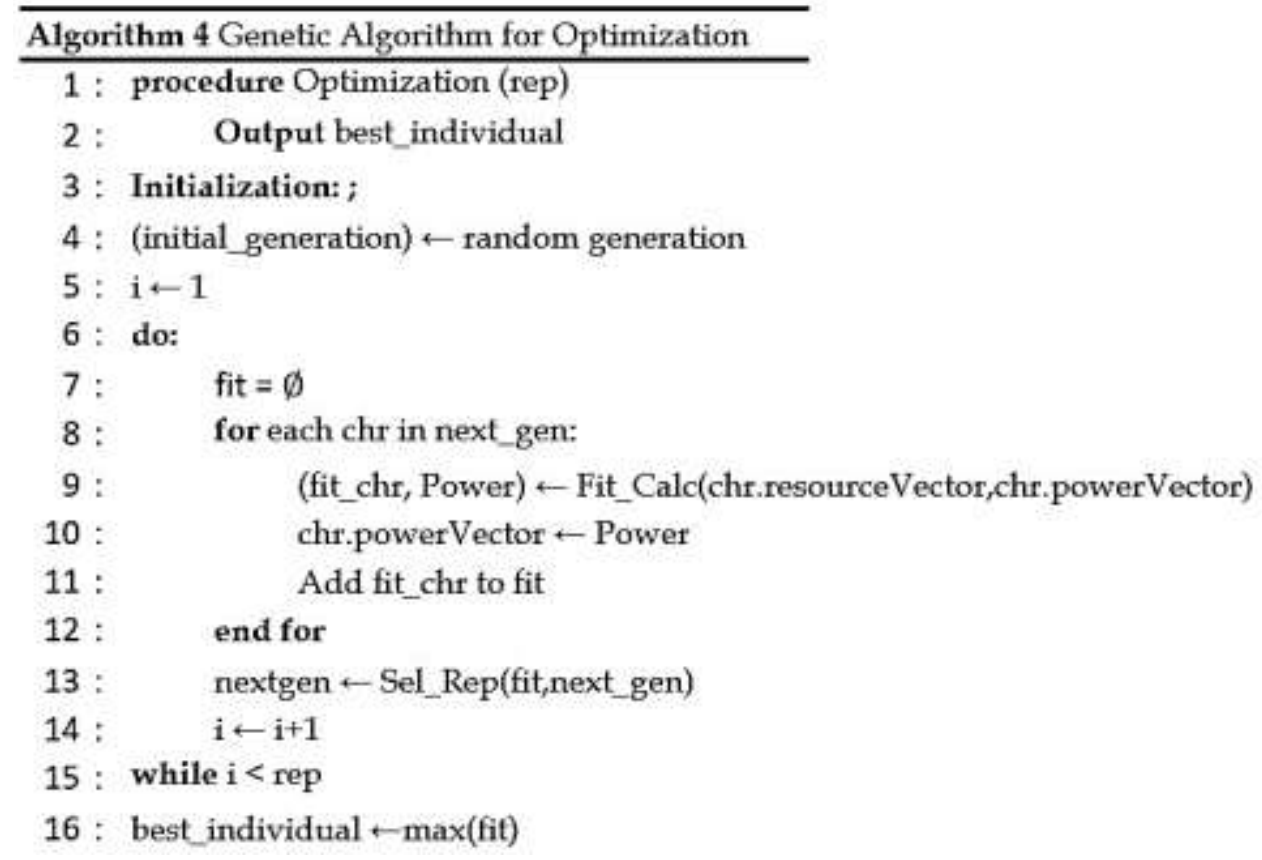

Figure 6. Genetic algorithm for optimization 


\subsubsection{Optimization using genetic algorithm}

The steps of this process are explained as follows. At first, $\mathrm{N}_{\mathrm{t}}$ random generation is generated as the first generation. The fitness value for first generation is also calculated with the maximum power of each user. Then, select and reproduce process will be executed to selects the parents and produce offspring. Next the mutation process will take place on the offspring. This process will be repeated several times to calculate the best allocation in the system. The pseudocode of this process can be seen on Figure 6 .

\subsection{Greedy algorithm}

The Greedy Algorithm is an algorithm that considers the data rate received by cellular users and D2D pairs. The greedy algorithm allocation process begun with, the first D2D pair chose the RB that belongs to the CU, where there was the highest total data rate on an RB. After the D2D pair selected one of the CUE devices, the other D2D pair couldn't select the RB that belongs to that CUE. This process was repeated until all D2D pairs had an RB. Figure 7 shows the pseudocode of the greedy algorithm.

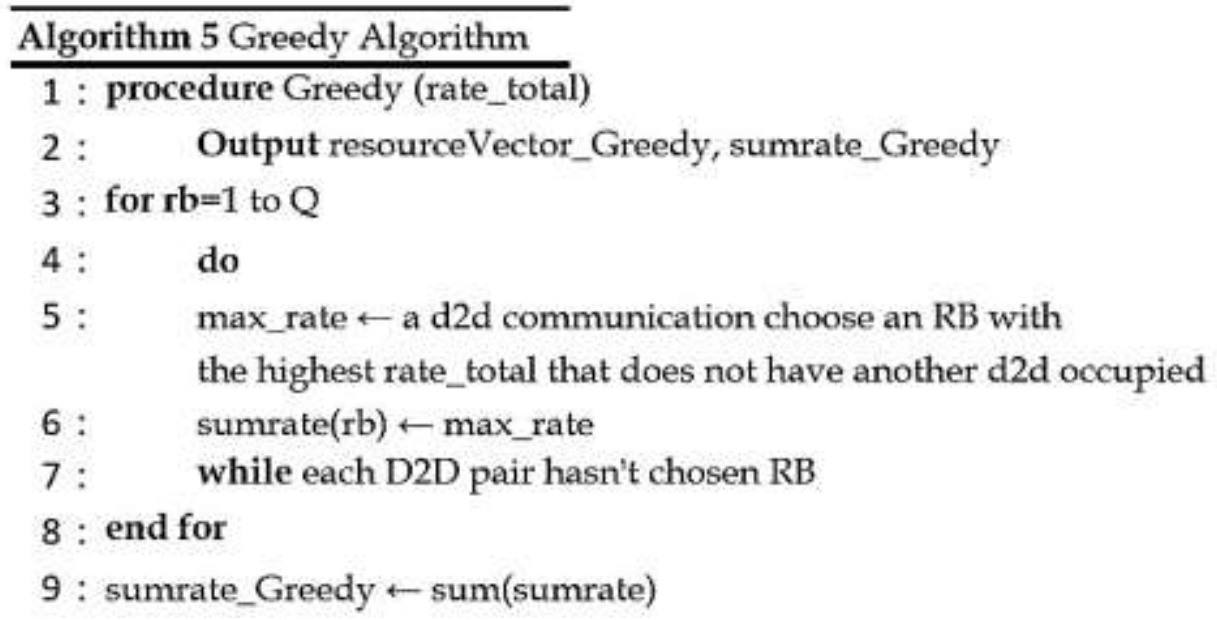

Figure 7. Greedy algorithm

\section{SIMULATION RESULTS AND ANALYSIS}

In this section, the performances of the Genetic algorithms are analyzed and compared with greedy algorithm. Several parameters that being observed are system sum-rate, system spectral efficiency, total power usage, system energy efficiency, and network interference level.

\subsection{Varied Number of Repetition}

At first, the genetic algorithm will be simulated independently with varies number of repitition. This simulation will observe the system's spectral efficiency in accordance with the original purpose. On this scenario, there are 10 CUEs and 10 DUEs on the cell. The results can be seen on Figure 8 and Table 2. 
Table 2. Spectral efficiency of genetic algorithm

\begin{tabular}{|c|c|c|}
\hline No. & Repetition & Spectral Efficiency (bps/Hz) \\
\hline 1 & 1 & 20.9845 \\
\hline 2 & 20 & 21.7975 \\
\hline 3 & 40 & 22.1319 \\
\hline 4 & 60 & 22.3495 \\
\hline 5 & 80 & 22.5275 \\
\hline 6 & 100 & 22.5938 \\
\hline 7 & 120 & 22.6758 \\
\hline 8 & 140 & 22.7462 \\
\hline 9 & 160 & 22.8128 \\
\hline 10 & 180 & 22.8464 \\
\hline 11 & 200 & 22.8940 \\
\hline
\end{tabular}

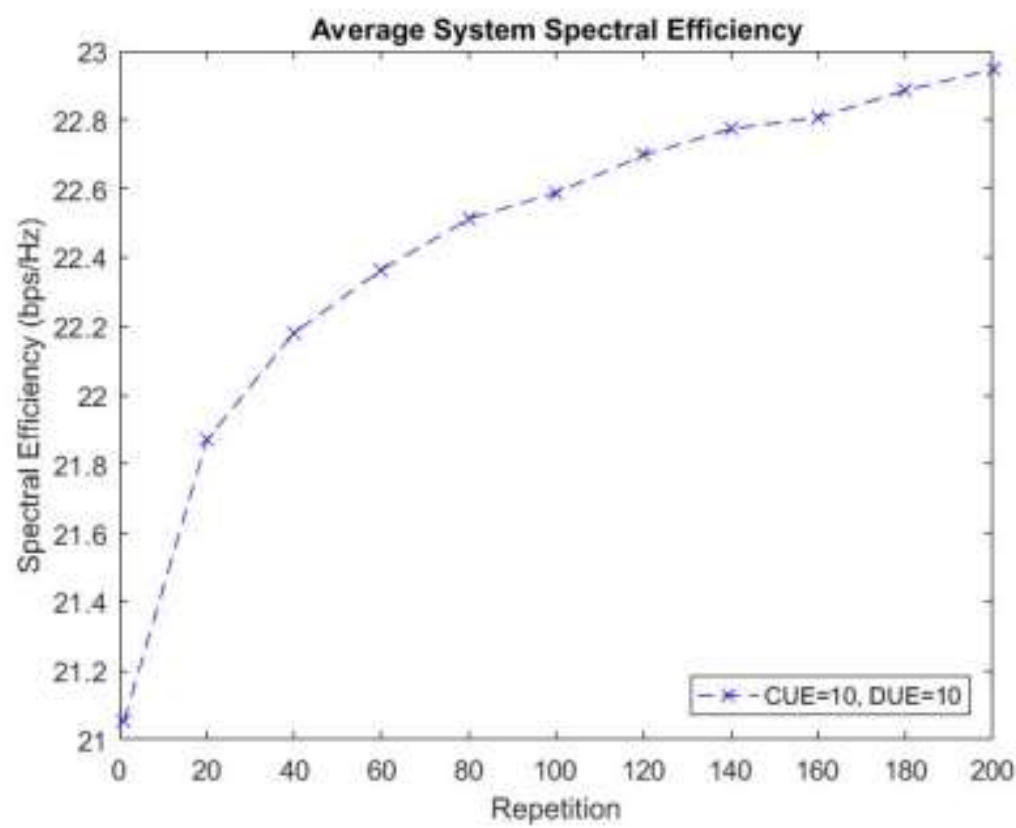

Figure 8. System spectral efficiency for different number of repetitions

From the results can be seen that, with increased number of repetitions, the spectral efficiency of the system increased too. But with more repetitions, the increment of the spectral efficiency is decline. This means the algorithm have found the solution for the optimization problem. The select, reproduce, and mutation that happened on the algorithm will not have much effect when the solution is found.

For the next simulation, there will be 3 different algorithm that being observed. The greedy algorithm, genetic algorithm with only one repetition, and genetics algorithm with 100 times repetition. This scenario was made to give a better comparison among the algorithm. On the next scenario, there will be 10 CUEs on the cell with varies number of DUEs. 


\subsection{Sumrate}

High system sum-rate will give a high data transmission speed so users can get more data. Simulation results on average system sumrate can be seen on Figure 9 and Table 3. The system sumrate will increase in line with the increasing number of DUE. The greedy algorithm is the best in this parameter. Greedy algorithm can achieve the average value of $45.36 \mathrm{Mbps}$. This happens because on greedy algorithm there are no power control scheme, so all user will get maximum power to communicate.

The genetic shows that the repetition really improve the algorithms performances. With 1 repetition, genetic algorithm only achieves $36.73 \mathrm{Mbps}$, while with 100 repetition this average number steps up to $39.97 \mathrm{Mbps}$. However, on 10 DUEs, the sumrate of 100 repetition genetics algorithm have best sumrate among the algorithm.

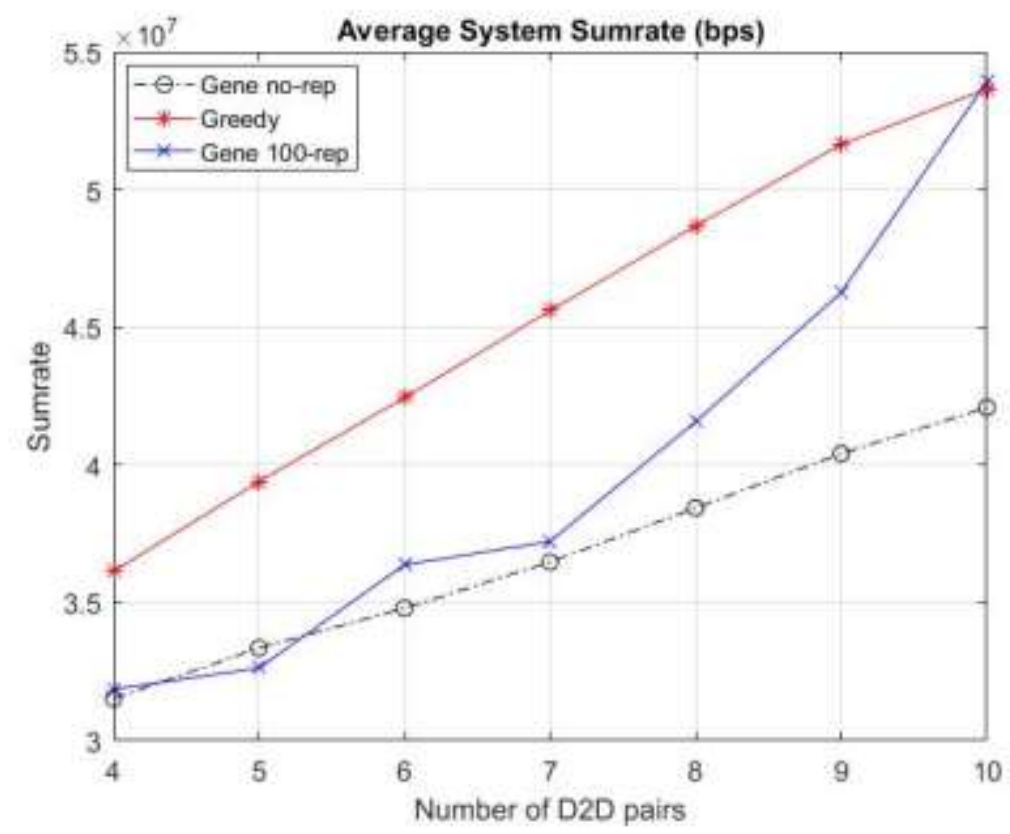

Figure 9. Sumrate of the system with varies number of DUEs

Table 3. Sumrate of the system

\begin{tabular}{|c|c|c|c|}
\hline \multirow{2}{*}{$\begin{array}{c}\text { Number of } \\
\text { DUE pairs }\end{array}$} & \multicolumn{3}{|c|}{ Sum-rate (x 107 bps) } \\
\cline { 2 - 4 } & $\begin{array}{c}\text { Genetic algorithm } \\
\text { 1x repetition }\end{array}$ & $\begin{array}{c}\text { Greedy } \\
\text { algorithm }\end{array}$ & $\begin{array}{c}\text { Genetic algorithm } \\
\text { 100 repetition }\end{array}$ \\
\hline 4 & 3.1522 & 3.6141 & 3.1864 \\
\hline 5 & 3.3358 & 3.9402 & 3.2635 \\
\hline 6 & 3.4806 & 4.2445 & 3.6381 \\
\hline 7 & 3.6482 & 4.5608 & 3.7225 \\
\hline 8 & 3.8445 & 4.8678 & 4.1578 \\
\hline 9 & 4.0415 & 5.1634 & 4.6266 \\
\hline 10 & 4.2088 & 5.3647 & 5.3907 \\
\hline Mean & 3.6731 & 4.5365 & 3.9979 \\
\hline
\end{tabular}




\subsection{Spectral Efficiency}

Simulation results on average spectral efficiency of each algorithm can be seen on Figure 10 and Table 4 . Same as the sumrate, spectral efficiency increases in line with the increasing number of DUE. The greedy algorithm is the best in this parameter. Greedy algorithm can achieve the average value of $22.68 \mathrm{bps} / \mathrm{Hz}$. This happens because on greedy algorithm there are no power control scheme, so all user will get maximum power to communicate. This result also caused by the greedy algorithm's constraint that limit the user on each RB with onle 1 CUE and 1 DUE pair, so the SINR value can be maintained.

The genetic shows that the repetition really improve the algorithms performances. With 1 repetition, genetic algorithm only achieves 18.36 bps $/ \mathrm{Hz}$, while with 100 repetition this average number steps up to 19.78 bps/Hz. But overall, genetic algorithm with 100 repetitions will have more complexity because of the repetition itself, while the other algorithm only uses 1 loop of the process.

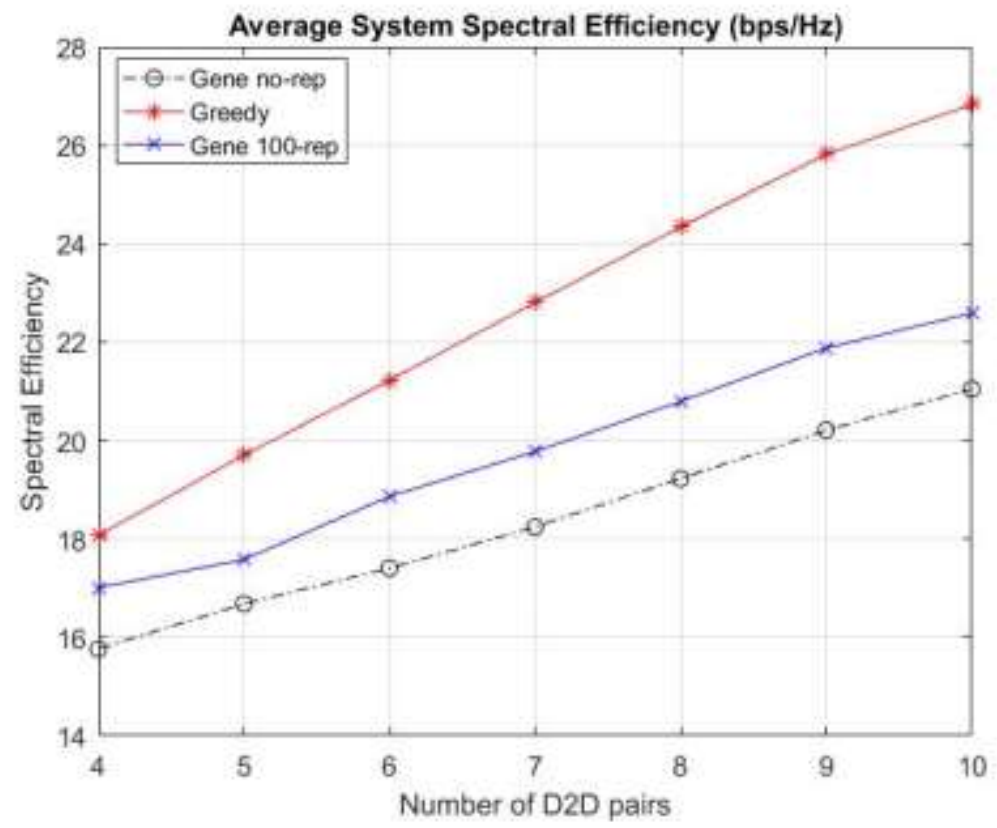

Figure 10. Spectral efficiency of the system with varies number of DUEs

Table 4. Spectral efficiency of the system

\begin{tabular}{|c|c|c|c|}
\hline \multirow{2}{*}{$\begin{array}{l}\text { Number of } \\
\text { DUE pairs }\end{array}$} & \multicolumn{3}{|c|}{ Spectral efficiency (bps/Hz) } \\
\hline & $\begin{array}{c}\text { Genetic algorithm } \\
1 \text { repetition }\end{array}$ & $\begin{array}{l}\text { Greedy } \\
\text { algorithm }\end{array}$ & $\begin{array}{c}\text { Genetic algorithm } \\
100 \text { repetition }\end{array}$ \\
\hline 4 & 15.7609 & 18.0707 & 17.0057 \\
\hline 5 & 16.6788 & 19.7012 & 17.5785 \\
\hline 6 & 17.4031 & 21.2227 & 18.8623 \\
\hline 7 & 18.241 & 22.8039 & 19.7728 \\
\hline 8 & 19.2223 & 24.3389 & 20.7919 \\
\hline 9 & 20.2076 & 25.8169 & 21.8784 \\
\hline 10 & 21.0441 & 26.8233 & 22.5877 \\
\hline Mean & 18.3654 & 22.6825 & 19.7825 \\
\hline
\end{tabular}




\subsection{Total Power Usage}

Total power usage in the system is calculated by summing all power transmitted in the system, including CUEs and DUEs. The simulation results can be seen on Figure 11 and Table 5. From Figure 11, can be concluded that the genetics algorithm with 100 repetitions have the lowest power usage among all algorithm.

Overall, all algorithm power usage increase in line with increasing number of DUEs. But in genetic algorithm, the power usage is more controlled. This happens because in the initial steps in genetic algorithm, there are power control scheme. The power control scheme limits the power transmit on all users. On 1 repetition, average total power usage is $2.17 \mathrm{~W}$. The repetition will decrease this number again, because in each repetition this usage power will be maximized to achieve better spectral efficiency on each RB. Meanwhile, the greedy algorithm, which use fixed power, the increasing number of users will increased the total power usage linearly.

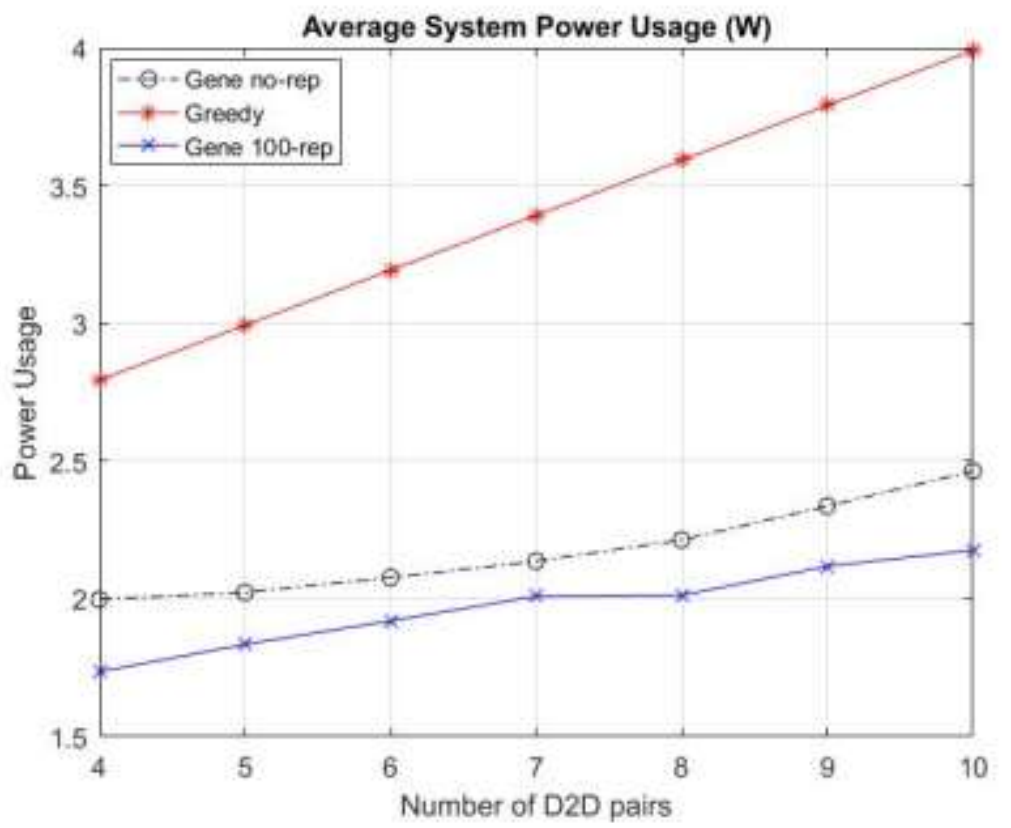

Figure 11. Total power usage in the system with varies number of DUEs

Table 5. Total power usage

\begin{tabular}{|c|c|c|c|}
\hline \multirow{2}{*}{$\begin{array}{c}\text { Number of } \\
\text { DUE pairs }\end{array}$} & \multicolumn{3}{|c|}{ Total power system (W) } \\
\cline { 2 - 4 } & $\begin{array}{c}\text { Genetic algorithm } \\
\text { 1 repetition }\end{array}$ & $\begin{array}{c}\text { Greedy } \\
\text { algorithm }\end{array}$ & $\begin{array}{c}\text { Genetic algorithm } \\
\text { 100 repetition }\end{array}$ \\
\hline 4 & 1.9975 & 2.7934 & 1.7355 \\
\hline 5 & 2.0234 & 2.9929 & 1.8347 \\
\hline 6 & 2.0773 & 3.1924 & 1.9200 \\
\hline 7 & 2.1364 & 3.3919 & 2.0092 \\
\hline 8 & 2.2137 & 3.5915 & 2.0127 \\
\hline 9 & 2.3366 & 3.791 & 2.1180 \\
\hline 10 & 2.4636 & 3.9905 & 2.1769 \\
\hline Mean & 2.1783 & 3.3919 & 1.9724 \\
\hline
\end{tabular}




\subsection{Energy Efficiency}

Energy efficiency means how many bit per second can be transferred in 1 Watt of power. The unit is bps/W (bit per second per Watt). The results of the simulation can be seen on Figure 12 and Table 6. In this parameters, genetic algorithm produce the best value. The 1 repetition genetic algorithm, the average energy efficiency is $17.07 \mathrm{Mbps} / \mathrm{W}$. This value jumps with 100 repetitions which achieve $20.27 \mathrm{Mbps} / \mathrm{W}$. Meanwhile greedy algorithm only achieve $13.35 \mathrm{Mbps} / \mathrm{W}$ in average.

This condition also happens caused by the power control scheme that included in the genetic algorithm. This scheme can minimize the power transmit on each user but still maximize the SINR on each RB. Greedy algorithm prove that with maximum transmitted power, the spectral efficiency can be maximized, but can be a waste in energy matters.

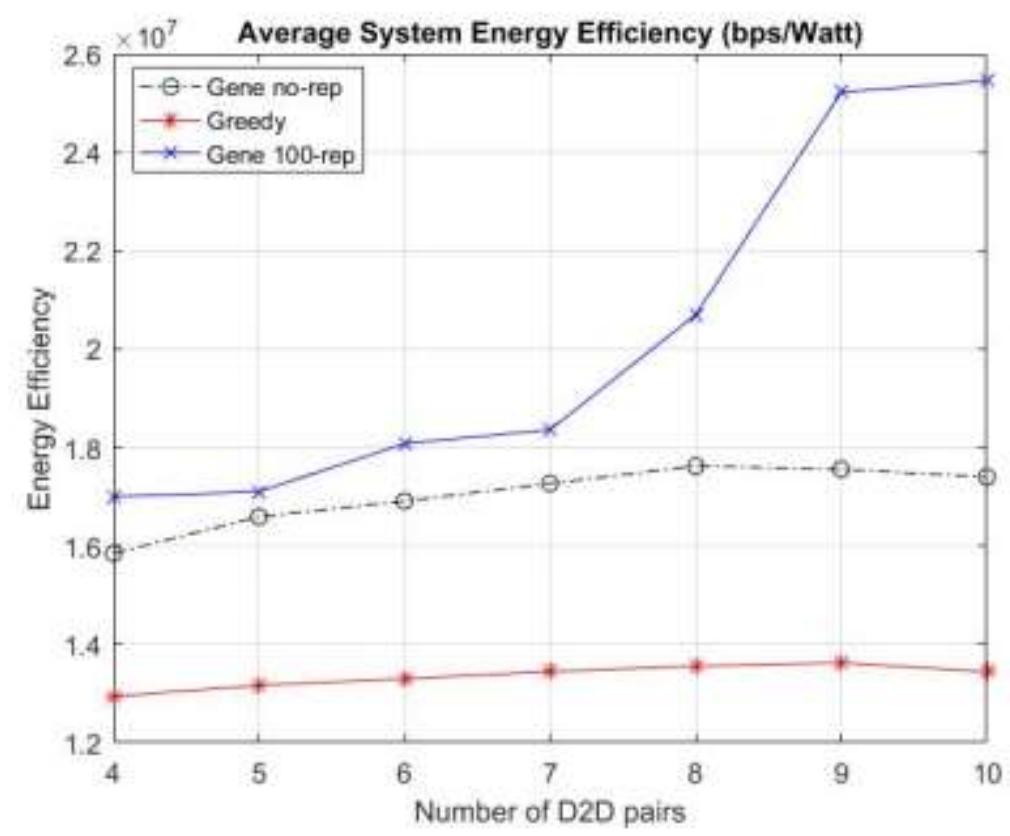

Figure 12. Energy efficiency of the system with varies number of DUEs

Table 6. Energy efficiency of the system

\begin{tabular}{|c|c|c|c|}
\hline \multirow{2}{*}{$\begin{array}{c}\text { Number of } \\
\text { DUE pairs }\end{array}$} & $\begin{array}{c}|c| \\
\text { Eenetic algorithm } \\
\text { 1 repetition }\end{array}$ & $\begin{array}{c}\text { Greedy } \\
\text { algorithm }\end{array}$ & $\begin{array}{c}\text { Genetic algorithm } \\
\mathbf{1 0 0} \text { repetition }\end{array}$ \\
\hline 4 & 1.5846 & 1.2938 & 1.6997 \\
\hline 5 & 1.6589 & 1.3165 & 1.7101 \\
\hline 6 & 1.6908 & 1.3296 & 1.8076 \\
\hline 7 & 1.7267 & 1.3446 & 1.8360 \\
\hline 8 & 1.7621 & 1.3554 & 2.0694 \\
\hline 9 & 1.7555 & 1.362 & 2.5217 \\
\hline 10 & 1.7398 & 1.3443 & 2.5452 \\
\hline Mean & 1.7026 & 1.3352 & 2.0271 \\
\hline
\end{tabular}




\subsection{Network Interference}

The total interference that happens in the cell on each algorithm can be seen on Figure 13 and Table 7. The genetic algorithm also shows the best result in this parameter. Genetic algorithm can achieve $-59.24 \mathrm{dBm}$ in average of interference level on 1 repetition, and can achieve a lower average interference which is $-98.40 \mathrm{dBm}$ with 100 repetition. Meanwhile the interference level on greedy algorithm only $-53.86 \mathrm{dBm}$ in average.

This result means the genetic algorithm can control and maintained the interference level in the cell. By decreasing and limiting each transmitted power in each user, the interference level on the system can stay at the lower level.

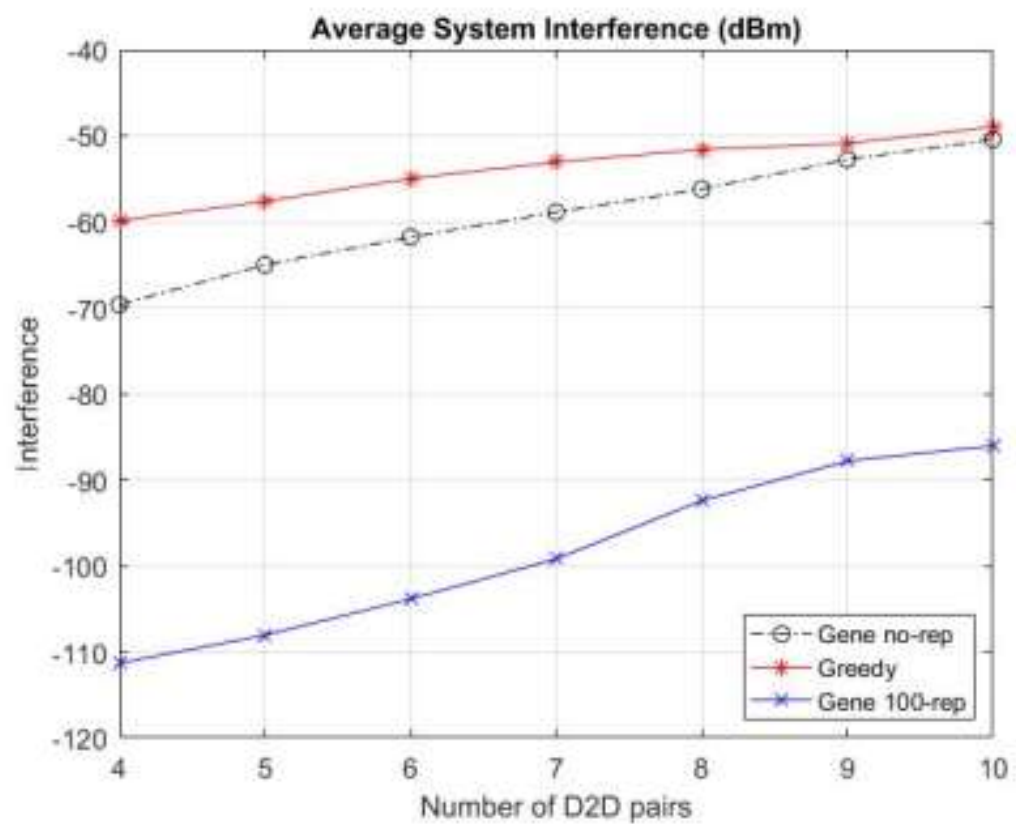

Figure 13. Network Interference with varies number of DUEs

Table 7. Network Interference

\begin{tabular}{|c|c|c|c|}
\hline \multirow{2}{*}{$\begin{array}{c}\text { Number of } \\
\text { DUE pairs }\end{array}$} & \multicolumn{3}{|c|}{ Network Interference (dBm) } \\
\cline { 2 - 4 } & $\begin{array}{c}\text { Genetic algorithm } \\
\text { 1 repetition }\end{array}$ & $\begin{array}{c}\text { Greedy } \\
\text { algorithm }\end{array}$ & $\begin{array}{c}\text { Genetic algorithm } \\
\text { 100 repetition }\end{array}$ \\
\hline 4 & -69.6518 & -59.8589 & -111.3231 \\
\hline 5 & -65.0128 & -57.6444 & -108.0780 \\
\hline 6 & -61.7879 & -54.9705 & -103.8633 \\
\hline 7 & -58.8895 & -53.0778 & -99.2212 \\
\hline 8 & -56.1939 & -51.5917 & -92.4305 \\
\hline 9 & -52.7546 & -50.9153 & -87.8322 \\
\hline 10 & -50.445 & -48.9822 & -86.0584 \\
\hline Mean & -59.2479 & -53.863 & -98.4010 \\
\hline
\end{tabular}


Table 8. Overall comparison among algorithm

\begin{tabular}{|c|c|c|c|}
\hline \multirow{2}{*}{ Parameter } & \multicolumn{3}{|c|}{ Algorithm's mean value } \\
\cline { 2 - 4 } & Greedy & Genetic 1 Repetition & Genetic 100 Repetitions \\
\hline Sumrate & $45.36 \mathrm{Mbps}$ & $36.73 \mathrm{Mbps}$ & $39.97 \mathrm{Mbps}$ \\
\hline Spectral Efficiency & $18.07 \mathrm{bps} / \mathrm{Hz}$ & $15.76 \mathrm{bps} / \mathrm{Hz}$ & $17.00 \mathrm{bps} / \mathrm{Hz}$ \\
\hline Energy Efficiency & $13.35 \mathrm{Mbps} / \mathrm{W}$ & $17.02 \mathrm{Mbps} / \mathrm{W}$ & $20.27 \mathrm{Mbps} / \mathrm{W}$ \\
\hline Power Usage & $3.39 \mathrm{Watt}$ & $2.17 \mathrm{Watt}$ & $1.97 \mathrm{Watt}$ \\
\hline Network Interference & $-53.86 \mathrm{dBm}$ & $-59.24 \mathrm{dBm}$ & $-98.40 \mathrm{dBm}$ \\
\hline
\end{tabular}

\subsection{Simulation Analysis}

Can be seen on the simulation results, Genetic algorithm has the exelence in energy efficiency, while greedy algorithm has a good spectral efficiency. The summaru of the simulation results can be seen on Table 8 , with the best value on each parameter is coloured grey.

The Greedy has more in sumrate and spectral efficiency because iit restrict each RB to only 2 users in one time maximum, which are 1 CUE and 1 DUE. The spectral efficiency can also be maximized by allocating maximum power on each user. But this schem caused a depression iin the energy efficiency and increasing interference among user in the system. This algorithm can cause a power wastage in the system.

Meanwhile in genetic algorithm, the power control and the repetition process maximized the energy efficiency and lowering the power usage and the interference level in the cell. By allocating each DUE according to the fitness of each RB, and allocating each user's power according to the condition of each RB, genetic algorithm can avoid power wastage in the system. In other hand, the spectral efficiency cannot be maximized. By increasing the repetition on the algorithm also makes the computational process on genetic algorithm requires more tima, so the system complexity also increase. These results mean there are trade-offs on among the algorithm.

\section{CONCLUSION}

In this research, the performances of genetic algorithm on D2D underlaying communication are analyzed, and then compared with the conventional greedy algorithm. The greedy algorithm allocates only based on the best SINR for each DUE, and allocates maximum fixed power on each user, without considering the other parameters. The results show that the greedy algorithm achieve higher value in sumrate and spectral efficiency, averaging $45.36 \mathrm{Mbps}$ and $18.07 \mathrm{bps} / \mathrm{Hz}$ respectively. The genetic algorithm only achieves an average sumrate at $39.97 \mathrm{Mbps}$ and average spectral efficiency at $17.00 \mathrm{bps} / \mathrm{Hz}$.

In the other hand, by allocating user and transmit power on each user with the purpose of maximizing the fitness value (in this case is spectral efficiency), genetic algorithm cannot achieve higher spectral efficiency compared to greedy algorithm. Although, genetic algorithm achieves better value in energy efficiency with average value in $20.27 \mathrm{Mbps} /$ Watt. The 
system's power usage also the lowest at 1.97 Watt, when greedy algorithm uses 3.39 Watt in average. The genetic algorithm also can maintain the system's interference level on $-98.40 \mathrm{dBm}$.

\section{REFERENCES}

[1] R. D. Ainul, H. Mahmudah, A. Wijayanti, Performance Analysis of Scheduling Schemes for Femto to Macro Interference Coordination in LTE-Femtocell Deployment Scenario, EMITTER International Journal of Engineering Technology, vol. 4, no. 11, pp 65-90, 2016

[2] Afif Osseiranetal, Scenarios for 5G mobile and wireless communications: The vision of the METIS project, IEEE Commun. Mag., vol. 52, no. 5, pp. 26-35, 2014.

[3] Li Wang, Huan Tang, Device-to-Device Communications in Cellular Networks, Springer International Publishing, 2016.

[4] Y. Priatama, Radio Resource Management and Interference Mitigation for Device to Device Communication Underlaying 5G Network, Undergraduated Thesis, Telkom University (Bandung), 2019.

[5] Guanding Yu, Lukai Xu, Daquan Feng, Rui Yin, Geoffrey Ye Li, Yuhuan Jiang, Joint mode selection and resource allocation for device-to-device communications, IEEE Trans. Commun., vol. 62, no. 11, pp. 38143824, 2014.

[6] Hengameh Takshi, Gulustan Dogan, Huseyin Arslan, Joint Optimization of Device to Device Resource and Power Allocation Based on Genetic Algorithm, IEEE Access, vol. 6, pp. 21173-83, 2018.

[7] V. S. W. Prabowo, B. Pamukti, A. Fahmi, N. M. Adriansyah and N. Andini, Joint-Greedy Allocation Algorithm on D2D Communication Underlaying Networks, 2019 IEEE Asia Pacific Conference on Wireless and Mobile (APWiMob), BALI, Indonesia, 2019, pp. 48-52.

[8] H. Ding, S. Ma, and C. Xing, 'Feasible D2D communication distance in D2D-enabled cellular networks,' in Proc. IEEE Int. Conf. Commun. Syst. (ICCS), Nov. 2014, pp. 1-5.

[9] K. Deb, Multi-Objective Optimization Using Evolutionary Algorithms, vol. 16. Hoboken, NJ, USA: Wiley, 2001. 